\title{
Legacy of Tunka-Rex software and data
}

P. Bezyazeekov,,${ }^{1, *}$ N. Budnev, ${ }^{1}$ O. Fedorov, ${ }^{1}$ O. Gress, ${ }^{1}$ O. Grishin, ${ }^{1}$ A. Haungs, ${ }^{2}$

T. Huege, ${ }^{2,3}$ Y. Kazarina, ${ }^{1}$ M. Kleifges, ${ }^{4}$ E. Korosteleva, ${ }^{5}$ D. Kostunin, $, 6,9$

L. Kuzmichev, ${ }^{5}$ V. Lenok, ${ }^{2}$ N. Lubsandorzhiev, ${ }^{5}$ S. Malakhov, ${ }^{1}$ T. Marshalkina, ${ }^{1}$

R. Monkhoev, ${ }^{1}$ E. Osipova, ${ }^{5}$ A. Pakhorukov, ${ }^{1}$ L. Pankov, ${ }^{1}$ V. Prosin, ${ }^{5}$

\section{F. G. Schröder, ${ }^{2,7}$ D. Shipilov ${ }^{8}$ and A. Zagorodnikov ${ }^{1}$}

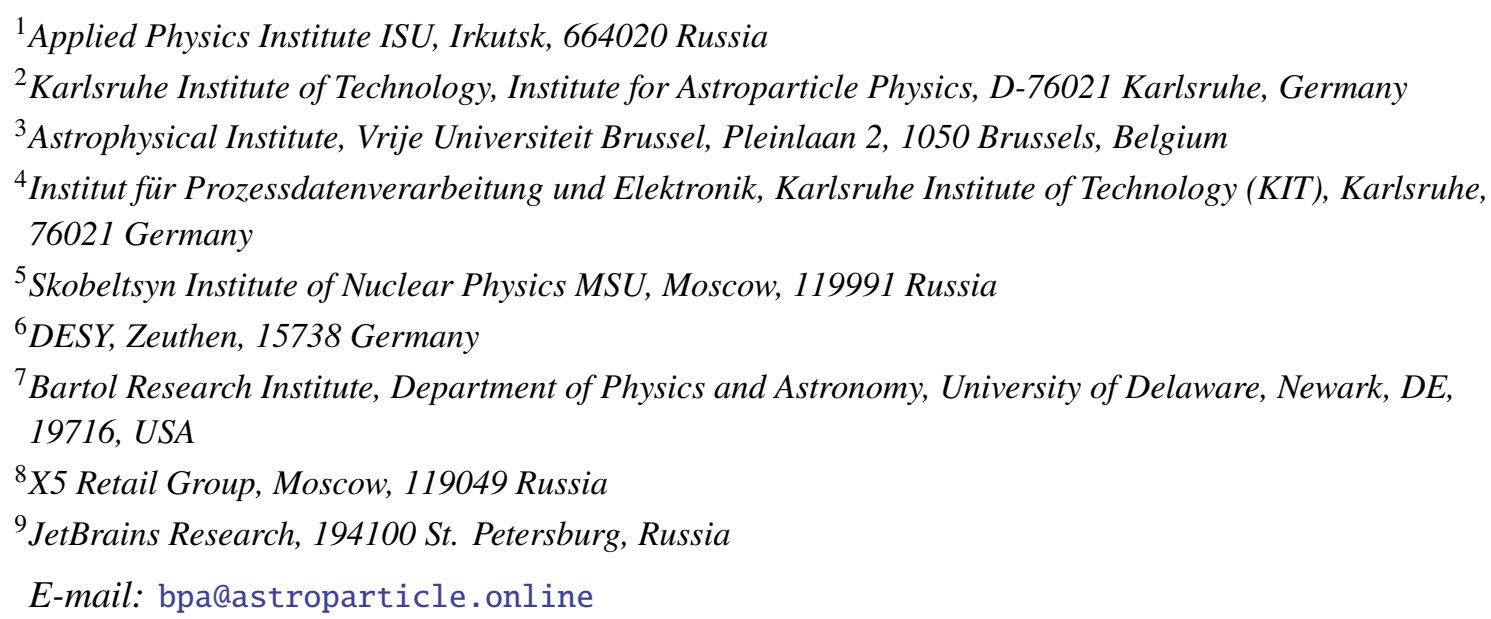

Tunka-Rex is a digital antenna array for measuring the radio emission from air-showers, induced by high-energy cosmic rays. The array started operation in 2012 with 18 antennas and had significantly developed over the years, finishing measurements in 2019 with 63 antennas and upgraded data acquisition. Analysis and processing of the collected data is a complex procedure which contains a number of steps (monitoring of state of the array, low-level filtration, quality cuts, reconstruction of air-shower parameters etc.). We give an overview of software developed for these tasks and our experience gained during the work with Tunka-Rex data. The legacy of software and data is discussed in the frame of the FAIR (Findability - Accessibility - Interoperability Reuse) concepts.

The 5th International Workshop on Deep Learning in Computational Physics 28-29 June, 2021

Moscow, Russia

\footnotetext{
${ }^{*}$ Speaker
} 


\section{Introduction}

Cosmic rays (CR) are high-energy charged particles moving in the space. Study of CR is important task in modern astrophysics because understanding of mechanisms of their generation and acceleration is necessary to understand the nature of high energy processes taking place in the Universe. Ultra-high energy CR (UHECR) are not available for direct measurements due to their low flux. However, when UHECR penetrates the atmosphere, it interacts with atmospheric particles and initiates the cascade process, namely extensive air-shower (EAS). EAS contains several components available for observation from ground level: hadrons, electrons, muons, wide-range electromagnetic emission, etc.

In comparison with other methods of EAS measurements, radio technique has specific advantages such as high duty cycle and cost-efficiency. However, radio method is sensitive to background conditions and dependent on complex procedures of data processing. For correct interpretation of measured data and precise reconstruction of primary cosmic ray parameters, one should to take into account the number of parameters like features of signal shape, efficiency of measurements, and background characteristics.

Tunka-Rex was a EAS radio detector operating in 2012-2019 [1]. During that time we collected a big dataset and developed software for processing the measured data, reconstruction the parameters of a primary particle, and handling the data in the framework of the Tunka-Rex Virtual Observatory [2]. This paper contains a brief summary of our experience gained with Tunka-Rex and description of the developed methods. Detailed descriptions can be found in the references.

\section{Template fitting method}

The standard method of the reconstruction of primary-particle energy and its mass, which was used at the first Tunka-Rex reconstruction, is based on the analysis of the lateral distribution of the measured EAS pulse amplitudes [3]. Using this approach we reach the precision of $15 \%$ for the energy and $38 \mathrm{~g} / \mathrm{cm}^{2}$ for $X_{\max }$ (depth of shower maximum) [4], which is directly related to the mass of primary particle. The precision is estimated by a comparison with the data-analysis results of the host experiment Tunka-133 (air-Cherenkov timing array) [5].

To improve the precision of reconstruction, we developed a new method taking into account the shape of the EAS pulse. For this study we prepared a library of EAS simulated signals initiated by nuclei with various masses. To define the primary-particle parameters, we concatenate the envelopes of EAS pulses of each measured event to single time series, as it is shown in Figure 1 (left). Then we perform a chi-square fit of this time series with simulated pulses normalized by amplitude and define the primary particle parameters by fit, as it is shown in Figure 1 (right).

Using this method with an additional signal pre-processing and taking into account systematic uncertainties caused by the atmospheric-refraction dynamics, we reached the precision of the energy reconstruction of $10 \%$ and the $X_{\max }$ reconstruction of $25-35 \mathrm{~g} / \mathrm{cm}^{2}$ (depends on the energy). A comparison of our reconstruction with the data from other experiments is shown in Figure 2. For the detailed description of the method see reference [6]. 

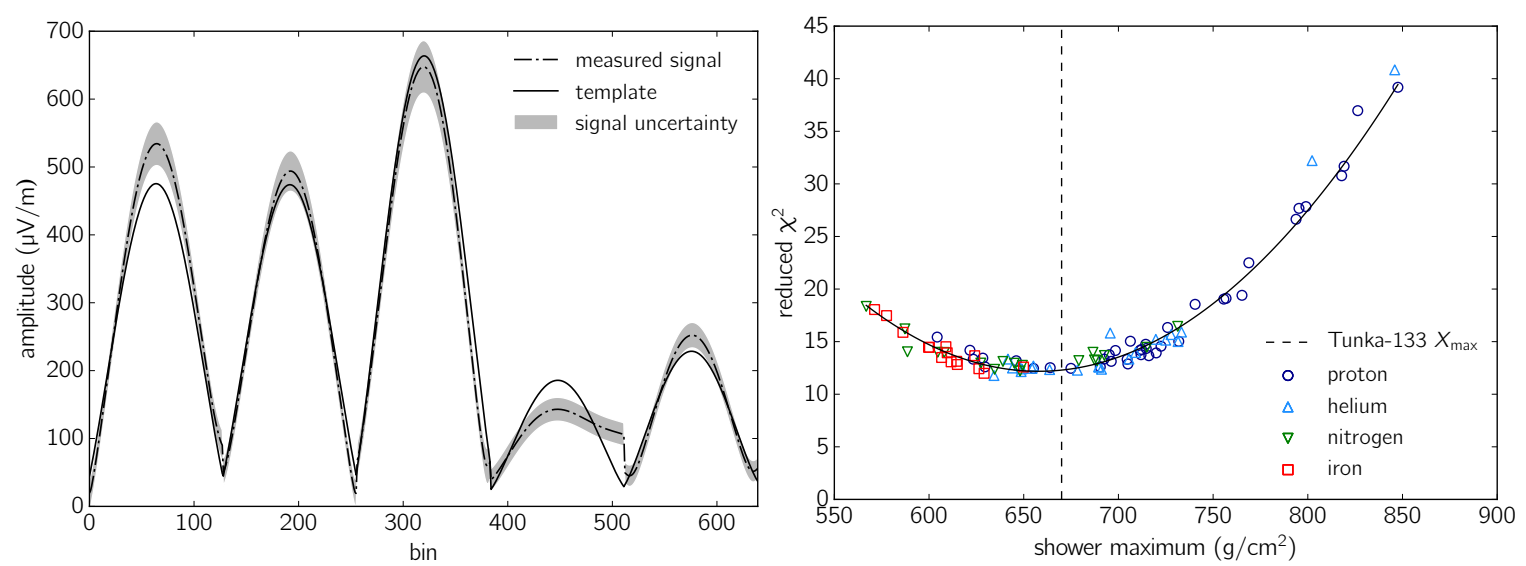

Figure 1: Left: Concatenated envelopes of the measured pulses from the different stations and scaled templates. The shape of the fourth signal is caused by a hardware artifact, however, it is possible to process it using this method. Right: A fitted $\chi^{2}$ distribution for the shower maximum reconstruction. The minimum of the fit represents reconstructed $\mathrm{X}_{\max }$. The dashed line represents $\mathrm{X}_{\max }$ reconstructed by the host experiment.

\section{Denoising using autoencoder}

Tunka-Rex works in low signal-to-noise ratio conditions. This fact leads to complications with processing the low-amplitude pulses from low-energy events up to impossibility of their reconstruction. To resolve this complications, we developed a reconstruction procedure for the low-energy events using pre-processing the signal traces by a convolutional autoencoder. In this procedure a 1-dimensional autoencoder with 3 convolutional layers in the encoding part removes the noise from the signal traces leaving only air-shower signal. Specifications of learning procedure and tests on simulated data is shown in reference [7]. After the tests on the simulated data, we developed a method of primary-particle energy reconstruction for validating the autoencoder approach with the measured data. For this study we chose a set of under-threshold low-energy events recorded with Tunka-Rex by the Tunka-133 triggering (energies from $10^{16}$ to $10^{17} \mathrm{eV}$ ). For this data we apply additional quality cuts on arrival direction. The chosen architecture requires a pre-normalisation of the input data, so, we lose information about the absolute amplitude of the signal. For handling this, we used approach defined as follows:

- Denoising the traces inside an event and collecting a timestamps of a peaks of the denoised pulses.

- Shifting the traces between each other corresponding to the difference between the peak timestamps.

- Applying the coherent sum of the given traces and obtaining a trace with the higher signalto-noise ratio.

Coherent sum method can be described as follows:

1. For each event we denoise the set of corresponding traces and find timestamps of denoised pulses. 


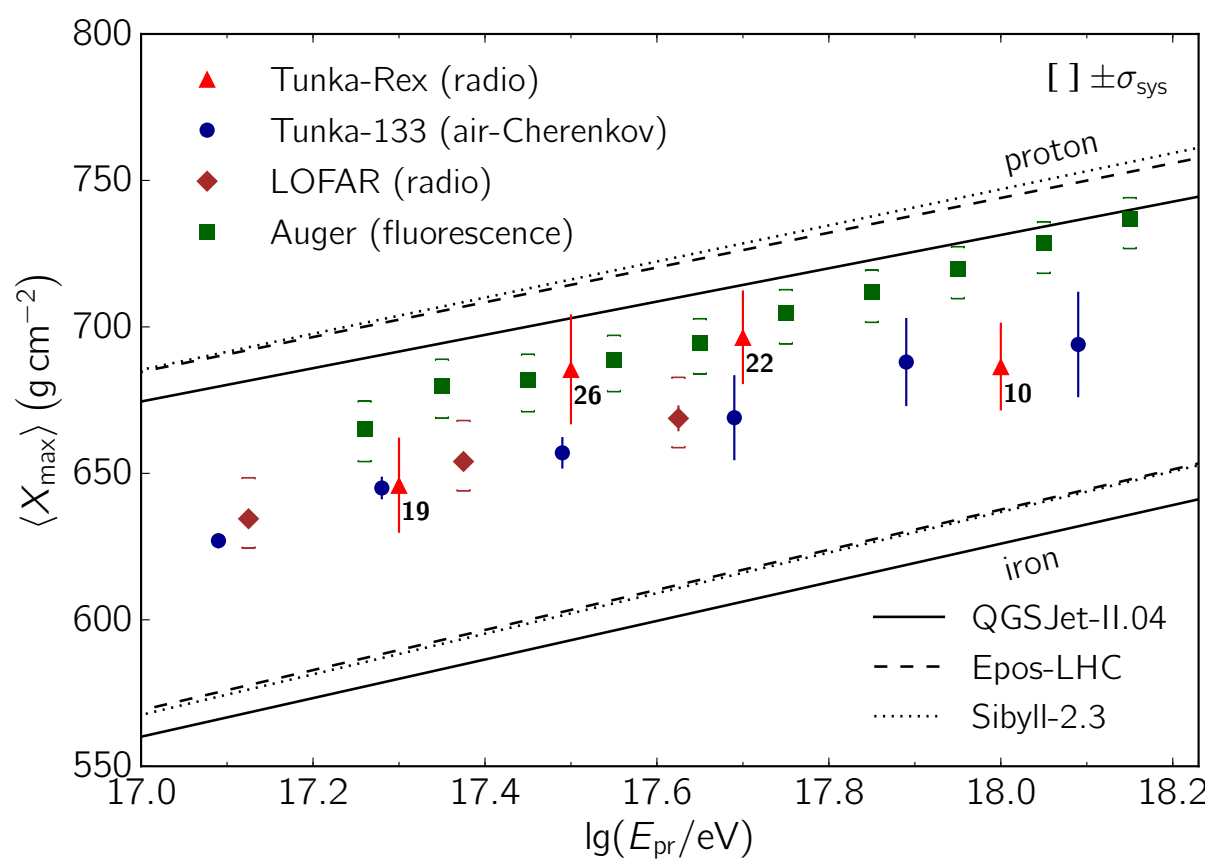

Figure 2: $X_{\max }$ reconstructed with the Tunka-Rex data using the template fit method in comparison to the reconstruction by the other experiments. The lines represent the mean values for proton and iron by different models.

2. We shift the recorded traces to match the founded timestamps.

3. We sum the shifted traces and obtain a trace with bigger SNR. The distance from "virtual station" corresponds to this trace to shower axis is calculated as a mean distance from the antennas with the signal to the shower axis.

With this method we obtained the traces with larger SNR than those in the initial traces and corresponding distances from the "virtual station" to shower axis. For further processing we select the "cohered" traces with resulting SNR > 16. After that we can reconstruct the primary particle energy using the single antenna method [8]. However, since this method is tuned for the high energies, we performed the data-driven re-calculation of the lateral-distribution function's (LDF) slope of the measured amplitudes. For this we fit the distribution of amplitudes vs distances obtained by the coherent sum method by a exponential function and find its slope (corresponding to mean slope for this energies) (see Figure 3).

Using the obtained slope $\eta$ we calculates the energy of the primary particle for selected events:

$$
E_{\mathrm{pr}}=\kappa E_{\mathrm{ALDF}} \exp \left[\left(d_{\mathrm{ALDF}}-d_{0}\right) \eta\right] / \sin \alpha,
$$

where $\kappa=868 \mathrm{EeV} /(\mathrm{V} / \mathrm{m}), d_{0}=180 \mathrm{~m}, \eta=0.00228$ and $\alpha$ is geomagnetic angle (the angle between shower axis and magnetic field of the Earth).

Using this method we reach the precision of the energy reconstruction $\approx 26 \%$ for the events with energies from $10^{16}$ to $10^{17} \mathrm{eV}$, which are not possible to reconstruct using the standard method. Comparison of the adaptive-LDF reconstruction and the host-experiment reconstruction is shown in Figure 4. For detailed description of the method see reference [9]. 

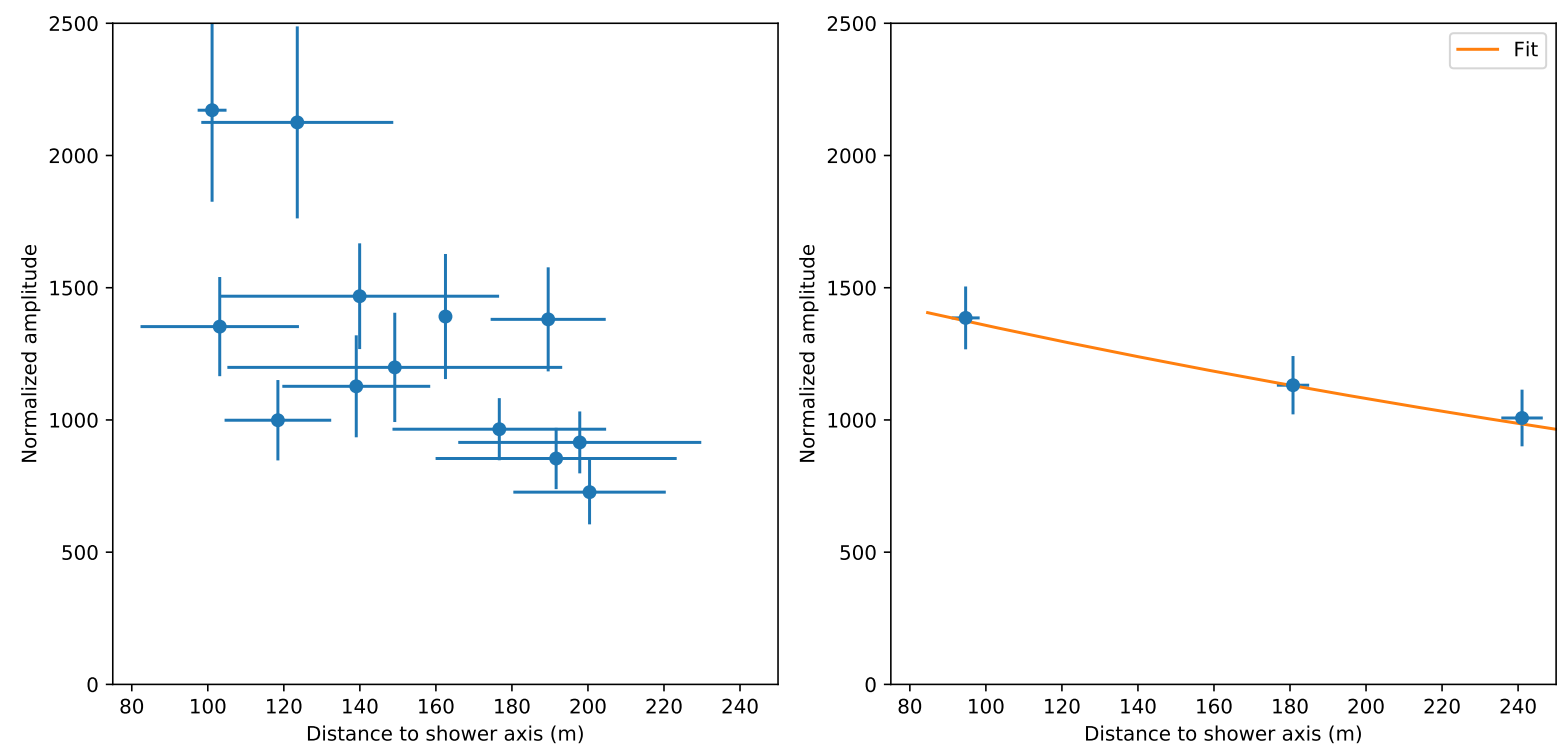

Figure 3: Left: The distribution of selected signals passed adaptive LDF processing. Error bars include uncertainties introduced by synthesis procedure. Right: An average LDF for the selected events. The orange curve indicates the exponential function fit, the slope of which is used for the energy reconstruction.
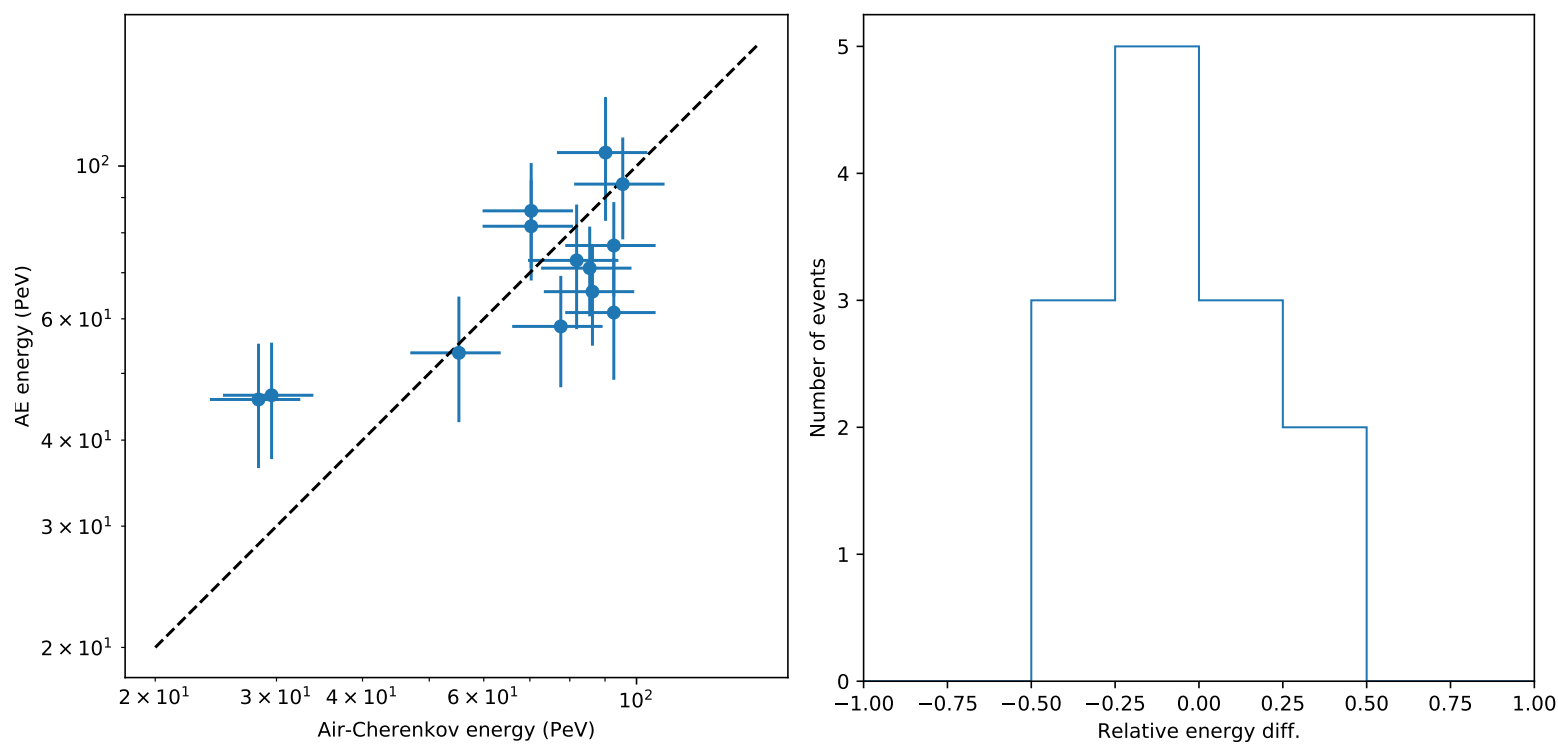

Figure 4: The performance of the energy reconstruction obtained for selected events. Left: cross-check with Tunka-133 reconstruction for the same events. Right: deviation between Tunka-133 and Tunka-Rex reconstruction showing Tunka-Rex resolution of $26 \%$.

\section{Tunka-Rex Virtual Observatory}

During the Tunka-Rex measurements we collected the huge set of data triggered by the Tunka133 and Tunka-Grande experiments. The lifecycle of this data is not ended with the experiment decommissioning. This data can be used for further analysis and testing the various methods of processing the astrophysical data. To provide an open access to our data and methods, we developed 
a framework named the Tunka-Rex Virtual Observatory (TRVO). For the time being, the framework is published online ${ }^{1}$ and contains a modular interface for accessing the set of low-level data (signal traces, timestamps and metadata) and processing this data using the methods developed by the Tunka-Rex group (includes creating subsets, statistical calculations, and autoencoder denoising). In the future we plan to deploy a high-level information includes the reconstruction and additional processing modules to TRVO. For the detailed description of TRVO see reference [10].

\section{Works based on Tunka-Rex experience and developments}

Using the experience gained during the work with Tunka-Rex, a number of studies were performed, such as implementation of the radio processing techniques in the context of multimessenger astrophysics [11], studying of the low-amplitude cosmological signal in the radio band using existing air-shower radio arrays [12], developing the self-trigger for an air-shower radio array [13, 14], applying the Tunka-Rex methods to the Almaty Radio Cluster [15], and adopting the method of autoencoder denoising to process the data from IceCube Surface Enhancement [16].

The most part of software we developed are placed in the Gitlab repository with an open access:

- Common libraries for data processing and analysis ${ }^{2}$

- Low-level data handling ${ }^{3}$, monitoring ${ }^{4}$, calibration ${ }^{5}$ and antenna software ${ }^{6}$ used in [17]

- Efficienty model $^{7}$ used in $[6,18]$

- Software used for the template method ${ }^{8}[6]$

- Denoiser based on autoencoder ${ }^{9}$, described in [9]

- SiMulation Manager (SiMM) software ${ }^{10}$, an interface for manipulation with large simulation libraries

- Tunka-Rex Virtual Observatory ${ }^{11}[19]$

\section{Conclusion}

Tunka-Rex is the first antenna array which successfully reconstructed the cosmic-ray energy spectrum and mass composition in the energy range $10^{17}-10^{18} \mathrm{eV}$. During the work with this detector we gained a lot of experience, developed a set of methods of data processing, and collected a huge

\footnotetext{
${ }^{1}$ https://jupyter.iap.kit.edu/user/doc/tree/tutorials/jbr/trvo/trvo_icrc.ipynb

2https://gitlab.ikp.kit.edu/tunkarex/pytrex

3https://gitlab.ikp.kit.edu/tunkarex/datatools

4https://gitlab.ikp.kit.edu/tunkarex/monitoring

5https://gitlab.ikp.kit.edu/tunkarex/calibration

6https://gitlab.ikp.kit.edu/tunkarex/antenna

7https://gitlab.ikp.kit.edu/tunkarex/efficiency

8https://gitlab.ikp.kit.edu/tunkarex/efieldfitter

${ }^{9}$ https://gitlab.ikp.kit.edu/tunkarex/denoiser

${ }^{10}$ https://gitlab.ikp.kit.edu/tunkarex/simm

${ }^{11}$ https://gitlab.ikp.kit.edu/tunkarex/trvo
} 
amount of data. We are going to provide an open access to all of that for the scientific community. We hope that it will be useful contribution for application in the wide range of astrophysical as well as radioastronomical purposes.

\section{Acknowledgments}

The work was performed at the UNU "Astrophysical Complex of MSU-ISU" (agreement 13.UNU.21.0007), and supported by the Russian Federation Ministry of Science and High Education (projects FZZE-2020-0017, FZZE-2020-0024).

\section{References}

[1] Tunka-Rex collaboration, Seven years of Tunka-Rex operation, PoS ICRC2019 (2020) 319 [1908.10305].

[2] TunKa-Rex collaboration, Tunka-Rex Virtual Observatory, PoS ICRC2021 (2021) 421.

[3] D. Kostunin, P.A. Bezyazeekov, R. Hiller, F.G. Schröder, V. Lenok and E. Levinson, Reconstruction of air-shower parameters for large-scale radio detectors using the lateral distribution, Astropart. Phys. 74 (2016) 79 [1504.05083].

[4] Tunka-Rex collaboration, Radio measurements of the energy and the depth of the shower maximum of cosmic-ray air showers by Tunka-Rex, JCAP 01 (2016) 052 [1509. 05652].

[5] V.V. Prosin et al. (Tunka-133 Collaboration), Tunka-133: Results of 3 year operation, Nucl.Instrum.Meth. A756 (2014) 94.

[6] TunKA-Rex collaboration, Reconstruction of cosmic ray air showers with Tunka-Rex data using template fitting of radio pulses, Phys. Rev. D97 (2018) 122004 [1803.06862].

[7] D. Shipilov et al., Signal recognition and background suppression by matched filters and neural networks for Tunka-Rex, .

[8] TUNKA-REX collaboration, Tunka-Rex: energy reconstruction with a single antenna station, EPJ Web Conf. 135 (2017) 01004 [1611.09614].

[9] Tunka-Rex collaboration, Reconstruction of sub-threshold events of cosmic-ray radio detectors using an autoencoder, PoS ICRC2021 (2021) 223 [2108. 04627].

[10] V. Lenok, D. Kostunin, O. Kopylova, P. Bezyazeekov, D. Wochele, F. Polgart et al., Tunka-Rex Virtual Observatory, PoS ICRC2021 (2021) 421 [2108. 04651].

[11] D. Kostunin, Radio detection in the multi-messenger context, EPJ Web Conf. 207 (2019) 03005 [1902.01776].

[12] D. Kostunin et al., Quest for detection of a cosmological signal from neutral hydrogen with a digital radio array developed for air-shower measurements, PoS ICRC2019 (2020) 320 [1908.06975]. 
[13] S. Malakhov, P. Bezyazeekov, O. Fedorov, Y. Kazarina, D. Kostunin and V. Lenok, Background identification algorithm for future self-triggered air-shower radio arrays, JINST 15 (2020) C09026 [2006. 03304].

[14] P. Bezyazeekov, O. Fedorov, Y. Kazarina, O. Kopylova, D. Kostunin, V. Lenok et al., Efficiency estimation of self-triggered antenna clusters for air-shower detection, PoS ICRC2021 (2021) 273 [2108.04642].

[15] S. Shinbulatov, N. Saduyev, D. Beznosko, O. Kalikulov, V. Zhukov, Y. Mukhamejanov et al., High-mountain hybrid installation for multicomponent detection of air-showers induced by ultra-high energy cosmic rays, PoS ICRC2021 (2021) 264.

[16] A. Rehman, A. Coleman, F.G. Schröder and D. Kostunin, Classification and Denoising of Cosmic-Ray Radio Signals using Deep Learning, PoS ICRC2021 (2021) 417.

[17] Tunka-Rex collaboration, Measurement of cosmic-ray air showers with the Tunka Radio Extension (Tunka-Rex), Nucl. Instrum. Meth. A802 (2015) 89 [1509. 08624].

[18] O. Fedorov et al., Detector efficiency and exposure of Tunka-Rex for cosmic-ray air showers, PoS ICRC2017 (2017) 387 [1712.00974].

[19] Tunka-Rex collaboration, Estimation of aperture of the Tunka-Rex radio array for cosmic-ray air-shower measurements, PoS ICRC2021 (2021) 210. 